\title{
A phase I, open-label, randomized crossover study to assess the effect of dosing of the MEK 1/2 inhibitor Selumetinib (AZD6244; ARRY-142866) in the presence and absence of food in patients with advanced solid tumors
}

\author{
Suzanne Leijen • Patricia M. M. B. Soetekouw • T. R. Jeffry Evans • \\ Marianne Nicolson • Jan H. M. Schellens • Maria Learoyd • Lynda Grinsted • \\ Victoria Zazulina · Thinn Pwint • Mark Middleton
}

Received: 9 March 2011 / Accepted: 24 August 2011/Published online: 28 September 2011

(C) The Author(s) 2011. This article is published with open access at Springerlink.com

\begin{abstract}
Purpose This Phase I study assessed whether food influences the rate and extent of selumetinib absorption in patients with advanced solid malignancies and determined the safety, tolerability, and pharmacokinetic (PK) profile of selumetinib and its active metabolite N-desmethyl-selumetinib in fed and fasted states.

Methods A single dose of $75 \mathrm{mg}$ selumetinib was to be taken with food on Day 1 followed by a single dose of $75 \mathrm{mg}$ after fasting for at least $10 \mathrm{~h}$ on Day 8, or vice versa, followed by twice daily dosing of $75 \mathrm{mg}$ selumetinib from Day 10 . Plasma concentrations and PK parameters were determined on Days 1 and 8. Patients could continue to receive selumetinib for as long as they benefitted from treatment.

Results In total, 31 patients were randomized to receive selumetinib; 15 to fed/fasted sequence and 16 to fasted/fed sequence. Comprehensive PK sampling was performed on 11 and 10 patients, respectively. The geometric least-squares means of $\mathrm{C}_{\max }$ and $\mathrm{AUC}$ for selumetinib were reduced by
\end{abstract}

\section{S. Leijen}

Division of Experimental Therapy, The Netherlands Cancer

Institute, Plesmanlaan 121, 1066 CX Amsterdam,

The Netherlands

\section{P. M. M. B. Soetekouw}

Division of Medical Oncology, Department of Internal Medicine, GROW-School for Oncology and Developmental Biology, Maastricht University Medical Centre, Maastricht,

The Netherlands

T. R. Jeffry Evans

Translational Cancer Research, Beatson West of Scotland

Cancer Centre, University of Glasgow, Glasgow G12 0YN, UK

M. Nicolson

Oncology Department, Aberdeen Royal Infirmary,

Foresterhill, Aberdeen, Grampian AB25 2ZN, UK
$62 \%$ (ratio $0.3890 \%$ CI $0.29,0.50$ ) and $19 \%$ (ratio $0.8190 \%$ CI $0.74,0.88$ ), respectively, under fed compared with fasting conditions. The rate of absorption $\left(\mathrm{t}_{\max }\right)$ of selumetinib (fed) was delayed by approximately $2.5 \mathrm{~h}$ (median). The food effect was also observed for the active metabolite N-desmethyl-selumetinib. Selumetinib was well tolerated.

Conclusions The presence of food decreased the extent of absorption of selumetinib. It is recommended that for further clinical studies, selumetinib be taken on an empty stomach. Selumetinib demonstrated an acceptable safety profile in the advanced cancer population.

Keywords MEK 1/2 inhibitor - Selumetinib - Phase I · Food effect

\section{Introduction}

Human cancers often arise as a result of mutations within components of cellular signaling pathways that coordinate

\section{J. H. M. Schellens $(\square)$}

Department of Clinical Pharmacology, and Division

of Experimental Therapy, The Netherlands Cancer Institute,

Plesmanlaan 121, 1066 CX Amsterdam, The Netherlands

e-mail: jhm@nki.nl

J. H. M. Schellens

Department of Pharmaceutical Sciences, Science Faculty, Utrecht University, Utrecht, The Netherlands

M. Learoyd · L. Grinsted · V. Zazulina

AstraZeneca, Alderley Park, Macclesfield,

Cheshire SK10 4TG, UK

T. Pwint $\cdot$ M. Middleton

Oxford NIHR Biomedical Research Centre,

Churchill Hospital, University of Oxford,

Oxford OX3 7LJ, UK 
cell proliferation, differentiation, and survival. The Ras/Raf/ mitogen-activated protein kinase (MEK)/extracellular signal-regulated kinase (ERK) pathway (Ras-Raf-MEK-ERK pathway) is an intracellular key signaling pathway that transmits mitogenic stimuli from the extracellular environment to the nucleus of the cell by multiple phosphorylation steps [1]. The cascade can be schematically divided into membranous and cytoplasmic phases. When growth factors bind to, and activate transmembrane receptors located on the cell surface, the level of guanosine triphosphate-bound forms of Ras increases in the cell [2, 3]. Upon Ras activation, Raf kinases are recruited to the plasma membrane and become activated. This process results in sequential phosphorylation and activation of several protein kinases, collectively known as the mitogen-activated protein kinase (MAPK) signaling cascade. In the MAPK cascade, Raf phosphorylates and activates MEK, which in turn phosphorylates and activates ERK. Activated ERK dissociates from the Ras/Raf/MEK/ERK complex and can translocate to the nucleus to phosphorylate and activate numerous different transcription factors [3-5]. MEK is the only enzyme known to activate ERK and being located downstream of Ras, and Raf has therefore been identified as an attractive therapeutic target within this central cascade. Inhibition of MEK activity is anticipated to inhibit tumor proliferation, differentiation, and survival. Given the widespread distribution of constitutive ERK activation, a MEK inhibitor could be foreseen to have a broad spectrum of clinical utility in both solid tumors and hematological malignancies.

Selumetinib $\left(\mathrm{C}_{17} \mathrm{H}_{15} \mathrm{BrClFN}_{4} \mathrm{O}_{3}\right.$; AZD6244 and ARRY142886) is a potent, selective, non-ATP competitive inhibitor of $\mathrm{MEK}$ with an $\mathrm{IC}_{50}$ of $14 \mathrm{nmol} / \mathrm{L}$ against purified MEK1 [6-8]. In contrast to its activity against MEK $1 / 2$, selumetinib did not show significant activity against various other kinases, enzymes, receptors, ion channels, and transporters. Selumetinib monotherapy has been clinically evaluated in a variety of cancers including melanoma, pancreatic, biliary tract, colorectal, and lung [9-13].

The initial formulation of selumetinib taken into Phase I was an extemporaneous preparation of an oral suspension of selumetinib, as the free base in an aqueous solution of sulphobutylether $\beta$-cyclodextrin $\left(\mathrm{Captisol}^{\circledR}\right.$ ), also referred to as selumetinib free base suspension or selumetinib "mix and drink formulation" [14]. Preclinical pharmacological studies revealed that this formulation showed good oral bioavailability at low doses, but there was dose-limited absorption with increasing dose (i.e. less than proportional increase), likely reflecting the low aqueous solubility of the free base. Selumetinib free base has a moderate permeability across Caco- 2 cells (an in vitro model of the human small intestinal mucosa used to predict in vivo drug absorption) and exhibits pH dependent solubility $(274 \mu \mathrm{g} / \mathrm{mL}$ at $\mathrm{pH} 1$ and $3.4 \mu \mathrm{g} / \mathrm{mL}$ at $\mathrm{pH} 7.4$; data on file). Because of these properties, selumetinib was classified as a tentative class IV compound (characterized by low permeability and low solubility).

For more convenient drug administration, and to achieve higher exposures, a capsule formulation of selumetinib as the hydrogen sulfate salt (AZD6244 Hyd-Sulfate) was developed, which was used in the present study [15]. The capsule formulation dissociates readily into sulfuric acid and selumetinib free base at equilibrium in water (data on file), with the capsule solubility measurements reflecting those of the free base suspension $(274 \mu \mathrm{g} / \mathrm{mL}$ at $\mathrm{pH} 1$, and $3.4 \mu \mathrm{g} / \mathrm{mL} \mathrm{pH}$ 7.4). The selumetinib capsule has two $\mathrm{pKa}$ values (acid/base ionization constants) at 2.7 and 8.2.

Excretion of selumetinib is predominantly via the feces. The majority of selumetinib metabolites were detected as glucuronide conjugates indicating that direct conjugation is a significant route of elimination for selumetinib. Selumetinib is metabolized by cytochrome P450 (CYPs) 1A2, 2C19, and 3A4, with CYP1A2 being responsible for the formation of the active metabolite, $\mathrm{N}$-desmethyl-selumetinib, which in vitro showed 3- to 5-fold greater potency for MEK1 inhibition than selumetinib itself. Another metabolite, selumetinib amide, is up to 50-fold less active than selumetinib and therefore unlikely to contribute significantly to biological activity.

Phase I studies with selumetinib free base suspension and capsule formulation showed that the pharmacokinetics (PK) was approximately dose proportional across the studied dose ranges for both formulations [15]. The maximal tolerated dose (MTD) for the free base suspension was $100 \mathrm{mg}$ BD and 75 BD for capsule formulation. Singledose $\mathrm{PK}$ revealed that selumetinib was absorbed relatively quickly at all dose levels with a median time to reach maximum plasma concentration $\left(\mathrm{t}_{\max }\right)$ of $1.5 \mathrm{~h}$ and a terminal elimination half-life $\left(\mathrm{t}_{1 / 2}\right)$ of $5-8 \mathrm{~h}$. Total body clearance of drug from plasma after an oral dose (CL/F, ranging from 12 to $23 \mathrm{~L} / \mathrm{h}$ ) and volume of distribution at steady state (Vss/F, ranging from 87 to $126 \mathrm{~L}$ ) also remained largely consistent across the studied dose range. Plasma N-desmethyl-selumetinib concentrations followed a similar PK profile, although exposure was much lower (AUC and $\mathrm{C}_{\max }$ generally $<15 \%$ of parent compound). Based on the dose-normalized area under the plasma concentration-time curve from time 0 to $24 \mathrm{~h}\left(\mathrm{AUC}_{0-24}\right)$, the estimated bioavailability of the capsule formulation was 2.5-fold higher than the free base suspension [15].

The current study was undertaken to investigate the effect of food on PK parameters of the capsule formulation, which was taken forward for further clinical development. It is known that food can influence the absorption of a drug by altering one or more of the manifold variables, ranging from postprandial changes of the gastrointestinal tract, e.g. delayed gastric emptying, to chemical characteristics of the 
drug itself, like modified solubility and changed metabolism [16-18]. Therefore, the present study was conducted to determine whether selumetinib should continue to be dosed on an empty stomach or whether it could be given with food.

\section{Methods}

\section{Aims}

The primary objective of this study was to assess whether food influenced the rate and extent of selumetinib absorption. Secondary objectives were to determine the PK of both selumetinib and $\mathrm{N}$-desmethyl-selumetinib in the presence and absence of food and to assess the safety and tolerability of selumetinib in patients with advanced solid malignancies.

An exploratory objective was included to assess the efficacy of selumetinib as measured by objective response rate (ORR) based on response evaluation criteria in solid tumors (RECIST 1.0) assessment in patients with measurable disease.

\section{Patient selection}

Male and female patients aged 18 years and over (World Health Organization (WHO)/Eastern Cooperative Oncology Group (ECOG) performance status 0-2) with advanced cancer, refractory to standard therapies or for whom no standard therapies existed, were enrolled in two investigational centers in the Netherlands and three in the United Kingdom. Patients had to be able to eat a high-fat breakfast within a 30-min time period. Required laboratory values consisted of absolute neutrophil count (ANC) $\geq 1,500$ per $\mathrm{mm}^{3}$, platelets $\geq 100,000$ per $\mathrm{mm}^{3}$, hemoglobin $>9.0 \mathrm{~g} / \mathrm{dL}$, serum bilirubin $<1.5 \times$ upper limit of normal (ULN), aspartate aminotransferase (AST) $<2.5 \times \mathrm{ULN}$, alanine aminotransferase (ALT) $<2.5 \times \mathrm{ULN}$, and calculated serum creatinine clearance $>50 \mathrm{~mL} / \mathrm{min}$ (using CockcroftGault formula or by 24-h urine collection).

Exclusion criteria included patients with refractory nausea and vomiting, chronic gastrointestinal diseases or significant bowel resection that would preclude adequate absorption; pregnant or lactating women; patients that received any radiotherapy or chemotherapy within 21 days prior to starting the study, or any investigational drug within the previous 28 days; patients with mean QTc (using Fridericia's correction) $>450 \mathrm{~ms}$ at screening or with factors that increased the risk of QT prolongation or arrhythmic events or patients using concomitant medication known to prolong QT interval; and patients with brain metastases or spinal cord suppression unless treated and stable for at least 1 month.

Informed written consent was obtained from all patients in accordance with federal and national guidelines, and the study was conducted in compliance with GCP guidelines and the Declaration of Helsinki. An additional informed written consent was needed for optional genetic blood sampling.

\section{Study design}

This was a Phase I, multi-center, open-label, randomized crossover study (NCT00710515). The dose of selumetinib was selected based upon the PK, safety, and tolerability data from the Phase I clinical study of the Hyd-Sulfate capsule formulation [15]. Eligible patients were randomized to one of two sequences. The first sequence received a single dose of $75 \mathrm{mg}$ selumetinib with food on Day 1, followed by a single dose of $75 \mathrm{mg}$ selumetinib in the fasted state on Day 8 . The second sequence received $75 \mathrm{mg}$ selumetinib in the fasted state (Day 1) and then $75 \mathrm{mg}$ selumetinib with food (Day 8) following a 7-day wash out period. Both groups continued on twice daily $75 \mathrm{mg}$ selumetinib from Day 10 onwards (extension period). Patients could continue to receive selumetinib until disease progression, unacceptable toxicity, or for as long as they continued to derive benefit from treatment. Selumetinib capsules were taken with approximately $240 \mathrm{~mL}$ of water. On Days 1 and 8, water could be taken freely up to $1 \mathrm{~h}$ prior to, and from $1 \mathrm{~h}$ after, dosing. In the fasted state, patients received no food or drink other than water for $10 \mathrm{~h}$ prior to dosing. In the fed state, patients were fed an FDA high-fat breakfast [19]. Patients were required to completely ingest this breakfast within $30 \mathrm{~min}$ and were dosed with a single oral $75 \mathrm{mg}$ dose of selumetinib $30 \mathrm{~min}$ after starting to eat breakfast. A standard meal was given at $4 \mathrm{~h}$ post-dose on both days, and until that time no food or drink (other than water) was received. For the BD dosing from Day 10 onwards, the extension period, the doses were taken $12 \mathrm{~h}$ apart. Both doses were taken in the fasted state. Breakfast could be taken from $1 \mathrm{~h}$ following dosing. Evening doses were not to be taken in the $1 \mathrm{~h}$ preceding a meal or in the $2 \mathrm{~h}$ after having finished a meal.

Treatment with selumetinib during the BD treatment phase was withheld if patients experienced an intolerable Adverse Event (AE) or any AEs $\geq$ Common Terminology Criteria (CTC) Grade 3. Selumetinib treatment could be restarted after the toxicity improved to a level considered by the Investigator to be manageable (i.e., CTC Grade 1, except for dermatological AEs where CTC Grade 2 was acceptable). Treatment could be resumed at the original dose or at a permanently reduced dose (50 mg selumetinib BD). 
Plasma sampling and assay methods

Blood samples were collected on Days 1 and 8, at pre-dose (within 30 min of dosing), 15 and $30 \mathrm{~min}$ and 1, 1.5, 2, 4, $8,12,24,36$, and $48 \mathrm{~h}$ post-dose for analysis of plasma selumetinib and N-desmethyl-selumetinib metabolite concentrations. For the analysis of selumetinib and N-desmethyl selumetinib in human plasma, solid phase extraction (SPE) was followed by high performance liquid chromatography (HPLC) with tandem mass spectrometric detection (MS/MS) with a calibration range that extended from 2 to $2,000 \mathrm{ng} / \mathrm{mL}$ for selumetinib and $2-500 \mathrm{ng} / \mathrm{mL}$ for $\mathrm{N}$-desmethyl selumetinib. ${ }^{13} \mathrm{C}_{6}$-selumetinib and ${ }^{13} \mathrm{C}_{6}-\mathrm{N}$ desmethyl selumetinib were used as internal standards in the assay. The analytes and internal standards were extracted from human plasma by SPE using Phenomenex Strata-X plates Polymeric Reversed Phase 96-well plates and injected onto a ultra performance liquid chromatography (UPLC) column (Acquity(tm) UPLC ${ }^{\circledR}$ BEH Phenyl, $1.7 \mathrm{~m}, 2.1 \times 50 \mathrm{~mm}$ ) or an HPLC column (Phenomenex Synergi, $4 \mu$ Polar-RP, $2.0 \times 50 \mathrm{~mm}$ ). The chromatographic separation and detection was achieved by liquid chromatography and tandem mass spectrometry (LC-MS/ MS) using a Sciex API5000, respectively. Selumetinib and $\mathrm{N}$-desmethyl selumetinib concentrations were determined by reference to calibration curves created by adding known concentrations of selumetinib and N-desmethyl selumetinib to control human plasma.

\section{Pharmacokinetic analysis}

Actual sample times were used for the PK analysis. All PK computations were performed at the Department of Clinical Pharmacology, Quintiles Overland Park, using WinNonlin Professional 5.2 and SAS ${ }^{\circledR}$ Version 8.2. To evaluate the PK characteristics of selumetinib and N-desmethylselumetinib in the presence and absence of food, the following parameters were determined by non-compartmental analysis: (1) For selumetinib: $\mathrm{C}_{\max }, \mathrm{t}_{\max }$, AUC, area under the plasma concentration-time curve from zero to the time of the last quantifiable plasma concentration $\left(\mathrm{AUC}_{0-\mathrm{t}}\right)$, terminal rate constant $\left(\lambda_{z}\right), t_{1 / 2}$, volume of distribution (apparent) during terminal $\left(\lambda_{\mathrm{z}}\right)$ phase $\left(\mathrm{V}_{\mathrm{z}} / \mathrm{F}\right)$ and $\mathrm{CL} / \mathrm{F}$. Though not a true indicator of rate, $\mathrm{t}_{\max }$ was used as an indicator of the effect of food on the rate of absorption of selumetinib. (2) For N-desmethyl selumetinib: $\mathrm{C}_{\max }, \mathrm{t}_{\max }$, $\mathrm{AUC}, \mathrm{AUC}_{0-\mathrm{t}}, \lambda_{\mathrm{z}}$, and $\mathrm{t}_{1 / 2}$.

\section{Assessments}

AEs were evaluated throughout the study and graded according to the Common Terminology Criteria for Adverse Events (CTCAE), version 3.0. In addition, the following safety assessments were performed: ophthalmological examination, ECGs, MUGA scan or echocardiogram, and safety laboratory evaluations. Tumor assessments, performed as exploratory analysis, were done every 6 -8 weeks, and Response Evaluation Criteria in Solid Tumors (RECIST 1.0) was used to assess response to treatment.

\section{Statistical evaluation}

At least 24 patients were to be randomized to the two sequences to assess whether the $90 \%$ confidence interval (CI) for the ratio of the food effect on AUC and $\mathrm{C}_{\max }$ lie entirely within the range of $(0.8,1.25)$. AUC and $\mathrm{C}_{\max }$ were $\log$ transformed and analyzed using an analysis of variance model allowing for the effect of food (fed or fasted), period, sequence, and patient within sequence. The adjusted geometric means (gls means) were estimated for each treatment arm (fed or fasted). An estimate of the food effect (ratio of the gls means of selumetinib in fed state: fasted state) was calculated together with its $90 \%$ CI. A formal test of carry-over was not performed. The potential for any carry-over was assessed by examination of pre-dose plasma concentrations on Day 8. An analysis of selumetinib $t_{\max }$ data was performed on untransformed data using a non-parametric analysis. The food effect, as measured by patient differences in $t_{\max }$ (fed minus fasted states), was analyzed using a Wilcoxon signed rank test. The HodgesLehmann estimator of median food effect was calculated and corresponding $90 \%$ CIs constructed.

The study included 3 main analysis populations: (1) the per protocol (PP) analysis set, (2) the Safety analysis set, and (3) the evaluable for PK analysis set. The PP analysis was to include all patients who were evaluable for PK analysis, had complied with the protocol requirements, and had no protocol violations. The Safety analysis set was to include all randomized patients who received at least 1 dose of study medication. Finally, the evaluable for PK analysis set was to include all randomized patients who had completed at least 1 period of the study and had sufficient PK data available to evaluate the primary outcome variables AUC and $C_{\max }$. The analysis of primary PK outcome variables was presented for both the group "evaluable for PK" and the group "per protocol (PP) analysis set". The PP analysis was considered primary for interpretation of data, with supportive interpretation from evaluable for PK analysis.

\section{Results}

Analysis sets and protocol deviations

Of 31 randomized patients, 30 were included into the PK and safety analysis set. One patient was excluded from the 
safety and PK set as she did not receive treatment. Nine patients were excluded from the PP analysis set due to protocol deviations. The following protocol deviations were observed: four patients had the drug administered more than $30 \mathrm{~min}$ after the breakfast; two patients did not complete both the fed and fasted periods of the crossover study; and five patients had insufficient breakfast consumption prior to dosing in the fed period.

Patient characteristics

Patient demographics and baseline disease characteristics are shown in Table 1 . The most commonly reported sites of primary tumor were skin/soft tissue tumors [melanoma ( 8 patients)], and colorectal (3 patients). Overall, metastatic disease was more commonly reported than locally advanced tumors with the majority of metastatic disease occurring at the following sites: lymph nodes, hepatic (including gall bladder), and respiratory. The largest proportion of patients $(36.7 \%)$ had received two prior chemotherapy regimens. This population was representative of typical Phase I population with pretreated patients.

Pharmacokinetics and bioavailability

Measurable concentrations of selumetinib were observed in both fed and fasted periods during the complete sampling period; up to 48 h (Fig. 1a). Administration of selumetinib on an empty stomach resulted in significantly higher selumetinib exposure: geometric least-squares means $\mathrm{C}_{\max }$ and AUC were reduced by $62 \%$ (ratio $0.3890 \%$ CI 0.29 , 0.50 ) and $19 \%$ (ratio $0.8190 \%$ CI $0.74,0.88$ ), respectively, under fed conditions, compared with exposure following $75 \mathrm{mg}$ of selumetinib taken fasted (Table 2; Fig. 2). The median $\mathrm{t}_{\max }$ of selumetinib $(75 \mathrm{mg})$ was delayed by approximately $2.5 \mathrm{~h}(90 \% \mathrm{CI} 1.77,3.03)$ in the presence of food. Results from a further $t_{\max }, A U C$ and $C_{\max }$ analysis conducted on data from all patients where PK data were

Table 1 Demographic characteristics

\begin{tabular}{|c|c|c|c|c|}
\hline \multicolumn{2}{|l|}{ Demographic characteristic } & \multirow{2}{*}{$\frac{\text { Fed/fasted }(N=15)}{9(60)}$} & \multirow{2}{*}{$\frac{\text { Fasted/fed }(N=15)}{12(80)}$} & \multirow{2}{*}{$\frac{\text { Total }(N=30)}{21(70)}$} \\
\hline Gender & Male & & & \\
\hline (n and \% of patients) & Female & $6(40)$ & $3(20)$ & $9(30)$ \\
\hline \multirow[t]{2}{*}{ Age (years) } & Mean (SD) & $59.7(9.01)$ & $56.6(10.25)$ & $58.1(9.61)$ \\
\hline & Range & $44 / 77$ & $32 / 70$ & $32 / 77$ \\
\hline Age group (years) & $\geq 18$ to $\leq 65$ & $12(80.0)$ & $13(86.7)$ & $25(83.3)$ \\
\hline ( $\mathrm{n}$ and $\%$ of patients) & $>65$ & $3(20)$ & $2(13.3)$ & $5(16.7)$ \\
\hline WHO performance status & 0 & $6(40.0)$ & $7(46.7)$ & $13(43.3)$ \\
\hline \multirow[t]{3}{*}{ ( $\mathrm{n}$ and \% of patients) } & 1 & $2(13.3)$ & $7(46.6)$ & $9(30.0)$ \\
\hline & 2 & $6(40.0)$ & 0 & $6(20.0)$ \\
\hline & $3-4$ & 0 & 0 & 0 \\
\hline \multirow{9}{*}{$\begin{array}{l}\text { Tumor site } \\
\text { (n and } \% \text { of patients) }\end{array}$} & Skin/soft tissue & 3 & 5 & $8(26.7)$ \\
\hline & Colon/colorectal/rectal & 3 & 3 & $6(20)$ \\
\hline & Head \& Neck & 1 & 1 & $2(6.7)$ \\
\hline & Esophagus & 1 & 1 & $2(6.7)$ \\
\hline & Biliary tract & 2 & 0 & $2(6.7)$ \\
\hline & Liver & 1 & 1 & $2(6.7)$ \\
\hline & Renal & 2 & 0 & $2(6.7)$ \\
\hline & Bladder & 1 & 1 & $2(6.7)$ \\
\hline & Other & 1 & 3 & $4(13.3)$ \\
\hline \multirow{4}{*}{$\begin{array}{l}\text { Number of prior regimens } \\
\text { ( } \mathrm{n} \text { and } \% \text { of patients) }\end{array}$} & 0 & $2(13.3)$ & $3(20.0)$ & $5(16.7)$ \\
\hline & 1 & $5(33.3)$ & $2(13.3)$ & $7(23.3)$ \\
\hline & 2 & $5(33.3)$ & $6(40.0)$ & $11(36.7)$ \\
\hline & $>2$ & $3(20.0)$ & $4(26.7)$ & $7(23.3)$ \\
\hline \multirow{4}{*}{$\begin{array}{l}\text { Race } \\
\text { (n and } \% \text { of patients) }\end{array}$} & Caucasian & $12(80)$ & $15(100)$ & $27(90)$ \\
\hline & Black & $1(6.7)$ & 0 & $1(3.3)$ \\
\hline & Asian-Non Japanese & $1(6.7)$ & 0 & $1(3.3)$ \\
\hline & Other & $1(6.7)$ & 0 & $1(3.3)$ \\
\hline
\end{tabular}



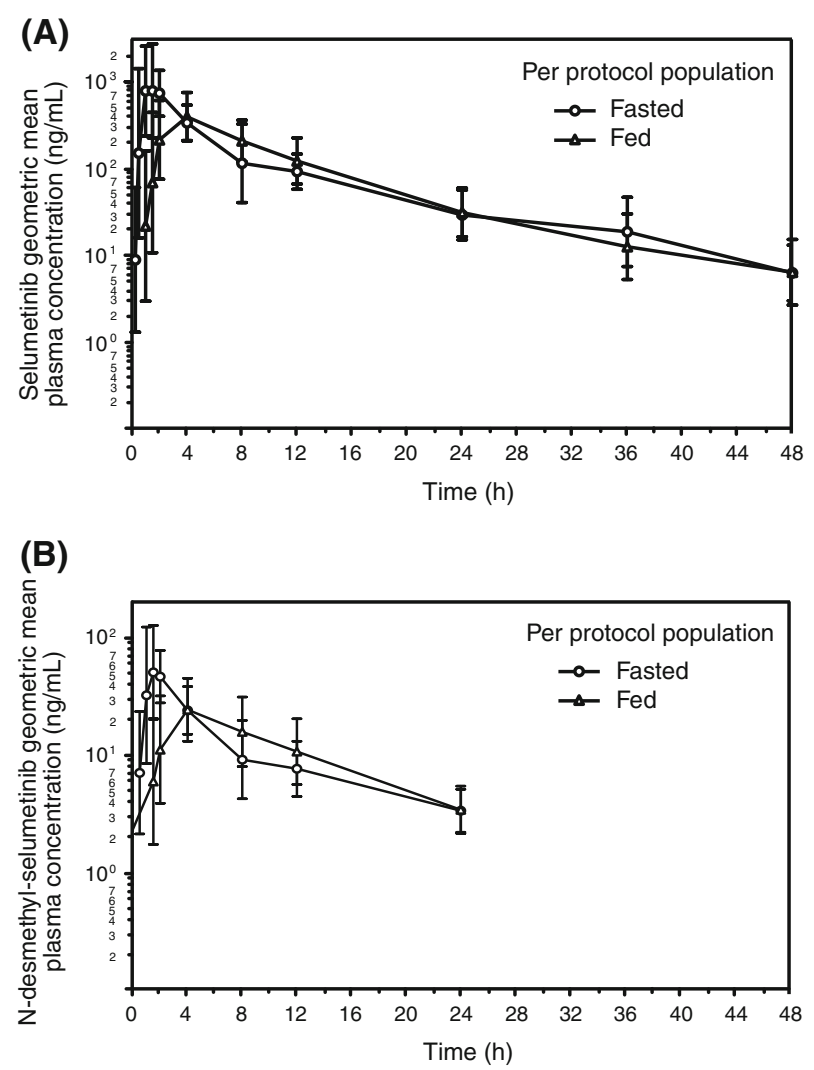

Fig. 1 Geometric mean $( \pm \mathrm{SD})$ concentration profiles of a selumetinib and $\mathbf{b} \mathrm{N}$-desmethyl-selumetinib in fed and fasted state (PP population)

evaluable were very similar to those observed in the PP population. The geometric mean clearance of selumetinib was lower (19\%) in the fasted state compared with the fed state reflecting the observed difference in AUC. The small difference in the volume of distribution $(\mathrm{Vz} / \mathrm{F})$ observed between the fasted and fed states did not translate into an appreciable difference in half-life (Table 2). Similar results were observed in the PK population.

Measurable concentrations of N-desmethyl-selumetinib were observed in both the fed and fasted periods for up to $24 \mathrm{~h}$ (Fig. 1b). Similar to selumetinib, the mean concentrations of N-desmethyl-selumetinib in the fed state were lower in comparison to the fasted state, and the $t_{\max }$ for $\mathrm{N}$-desmethyl-selumetinib was delayed when selumetinib was administered in the presence of food. A decrease of approximately $55 \%$ in the geometric mean $\mathrm{C}_{\max }$ and $9 \%$ in the geometric mean AUC of N-desmethyl-selumetinib was observed when selumetinib was given in the presence of food compared with the fasted state (Table 2). Whilst the presence of food reduced the exposure to selumetinib compared with the fasted state, the ratio of $\mathrm{N}$-desmethylselumetinib to selumetinib (metabolite to parent) remained similar $(0.06$ and $0.07 \mathrm{ng} * \mathrm{~h} / \mathrm{mL}$ for fed and fasted state, respectively). Hence, both the median plasma concentration of the parent compound and of the $\mathrm{N}$-desmethyl-metabolite were decreased in a similar extent when selumetinib was given in the presence of food. The median $t_{\max }$ for $\mathrm{N}$-desmethyl-selumetinib was delayed by approximately $3 \mathrm{~h}$ in the fed condition, also comparable to the delayed $t_{\max }$ of selumetinib.

Safety and tolerability

Of the 31 randomized patients, 28 completed the food effect period that involved 2 single doses of selumetinib (on Day 1 and Day 8) and continued on BD dosing from Day 10 for a mean duration of 58.7 days (median 52.0 days; range, 7-229 days). Two patients did not enter the extension period following voluntary discontinuation from the study. In the extension part, 10 patients had one or more dose reductions largely due to AEs $(n=6)$.

\section{Adverse events}

The most frequently reported AEs overall (regardless of severity, causality, or seriousness) were nausea, rash, diarrhea, fatigue, constipation, dyspnoea, and vomiting (Table 3). Eighteen out of $30(60.0 \%)$ patients (overall safety population) reported an $\mathrm{AE}$ of grade 3 or higher during the study, but only 4 (13.3\%) of whom had grade 3 AEs (Table 4) that were assessed as potentially treatment related. The $2(6.7 \%)$ grade 4 AEs were not considered to be causally related to study treatment (one patient experienced worsening of dyspnoea and one patient had hypercalcaemia). Serious AEs and AEs leading to discontinuation were reported by 40 and $20 \%$ of patients, respectively. One patient (3.3\%) died whilst on study as a result of an intestinal perforation due to disease progression. Although the case was reported as an AE leading to death, it was in fact due to disease progression. AEs that were only reported following chronic exposure to selumetinib in the extension phase were peripheral edema, hypertension, dry mouth, vision blurred, skin fissures, joint swelling, acne (acne/dermatitis acneiform), and dyspepsia. The most common AEs reported following single exposure to selumetinib were diarrhea, nausea, and abdominal pain.

Laboratory and vital sign findings in this study were generally consistent with those seen previously in selumetinib monotherapy studies. No notable changes in vital signs were observed.

\section{Anti-tumor activity/efficacy}

Although response evaluation was not the primary end point of this study, 28 patients were evaluable for objective response by RECIST. Two partial responses (7.1\%) were observed (Day 71 and on Day 189). Both patients had 
Table 2 Influence of food on the rate and extent of selumetinib absorption (A) and pharmacokinetic parameters for (B) selumetinib and (C) N-desmethyl-selumetinib in fed and fasted state [all PP population]

\begin{tabular}{|c|c|c|c|c|c|}
\hline \multirow[t]{2}{*}{ Selumetinib PK parameters } & \multirow[t]{2}{*}{ Treatment } & \multirow[t]{2}{*}{$n$} & \multirow[t]{2}{*}{ Geometric LS mean } & \multicolumn{2}{|c|}{ Pairwise comparision } \\
\hline & & & & Ratio of fed/fasted & $90 \% \mathrm{CI}$ \\
\hline \multicolumn{6}{|l|}{ (A) } \\
\hline \multirow[t]{2}{*}{ AUC (ng*h/mL) } & Fed & 21 & 4664 & & \\
\hline & Fasted & 19 & 5782 & 0.81 & $0.74,0.88$ \\
\hline \multirow[t]{2}{*}{$\mathrm{AUC}_{0-\mathrm{t}}(\mathrm{ng} * \mathrm{~h} / \mathrm{mL})$} & Fed & 21 & 4504 & & \\
\hline & Fasted & 21 & 5639 & 0.80 & $0.73,0.88$ \\
\hline \multirow[t]{2}{*}{$\mathrm{C}_{\max }(\mathrm{ng} / \mathrm{mL})$} & Fed & 21 & 557 & & \\
\hline & Fasted & 21 & 1450 & 0.38 & $0.29,0.50$ \\
\hline Selumetinib PK parameters & Treatment & $n$ & Geometric mean & $\mathrm{CV}(\%)$ & \\
\hline \multicolumn{6}{|l|}{ (B) } \\
\hline \multirow[t]{2}{*}{$t_{\max }(h)$} & Fed & 21 & $4.0^{\mathrm{a}}$ & $1.0,11.6^{\mathrm{a}}$ & \\
\hline & Fasted & 21 & $1.08^{\mathrm{a}}$ & $0.5,4.0^{\mathrm{a}}$ & \\
\hline \multirow[t]{2}{*}{$t_{1 / 2}(h)$} & Fed & 21 & 8.6 & 44.5 & \\
\hline & Fasted & 19 & 9.4 & 33.0 & \\
\hline \multirow[t]{2}{*}{ CL/F (L/h) } & Fed & 21 & 16.0 & 35.8 & \\
\hline & Fasted & 19 & 12.9 & 29.9 & \\
\hline \multirow[t]{2}{*}{$\mathrm{V}_{\mathrm{z}} / \mathrm{F}(\mathrm{L})$} & Fed & 21 & 199.4 & 56.2 & \\
\hline & Fasted & 19 & 175.2 & 46.8 & \\
\hline $\mathrm{N}$-desmethyl-selumetinib PK parameters & Treatment & $n$ & Geometric mean & $\mathrm{CV}(\%)$ & \\
\hline \multicolumn{6}{|l|}{ (C) } \\
\hline \multirow[t]{2}{*}{$\mathrm{C}_{\max }(\mathrm{ng} / \mathrm{mL})$} & Fed & 21 & 33.5 & 63.8 & \\
\hline & Fasted & 21 & 73.9 & 45.7 & \\
\hline \multirow[t]{2}{*}{$t_{\max }(h)^{a}$} & Fed & 21 & $4.0^{\mathrm{a}}$ & $1.0,11.6^{\mathrm{a}}$ & \\
\hline & Fasted & 21 & $1.50^{\mathrm{a}}$ & $0.5,4.0^{\mathrm{a}}$ & \\
\hline \multirow[t]{2}{*}{ AUC (ng*h/mL) } & Fed & 13 & 379.1 & 49.0 & \\
\hline & Fasted & 15 & 418.4 & 36.5 & \\
\hline \multirow[t]{2}{*}{$\operatorname{AUC}_{0-\mathrm{t}}(\mathrm{ng} * / \mathrm{h} / \mathrm{mL})$} & Fed & 21 & 282.4 & 68.2 & \\
\hline & Fasted & 21 & 376.4 & 41.0 & \\
\hline \multirow[t]{2}{*}{$\mathrm{t}_{1 / 2}(\mathrm{~h})$} & Fed & 13 & 6.8 & 56.6 & \\
\hline & Fasted & 15 & 7.7 & 45.5 & \\
\hline
\end{tabular}

${ }^{a}$ Data are presented as median (min, max)

cutaneous melanoma and were still alive and continuing study treatment at data cut-off. In addition, 5 (18\%) patients had the best overall response of stable disease, 2 for $>100$ days ( 1 colorectal cancer and 1 melanoma patient), 2 patients for $>50$ days ( 1 liver cancer and 1 prostate cancer).

\section{Discussion}

In this Phase I, multi-center, open-label, randomized crossover study, the influence of food on the rate and extent of selumetinib oral capsule absorption in male and female patients with solid malignancies was determined. In addition to selumetinib, concentrations of its 3- to 5-fold more active metabolite, $\mathrm{N}$-desmethyl-selumetinib, were also determined [15], as any changes in the metabolite concentrations may influence the treatment efficacy. The present study showed that the extent $\left(\mathrm{C}_{\max }\right.$ and AUC) and rate of absorption of selumetinib was significantly reduced in the presence of food. It is therefore recommended that for future clinical studies, selumetinib should continue to be administered on an empty stomach (no food or drink other than water for $2 \mathrm{~h}$ prior to dosing and $1 \mathrm{~h}$ after dosing). 

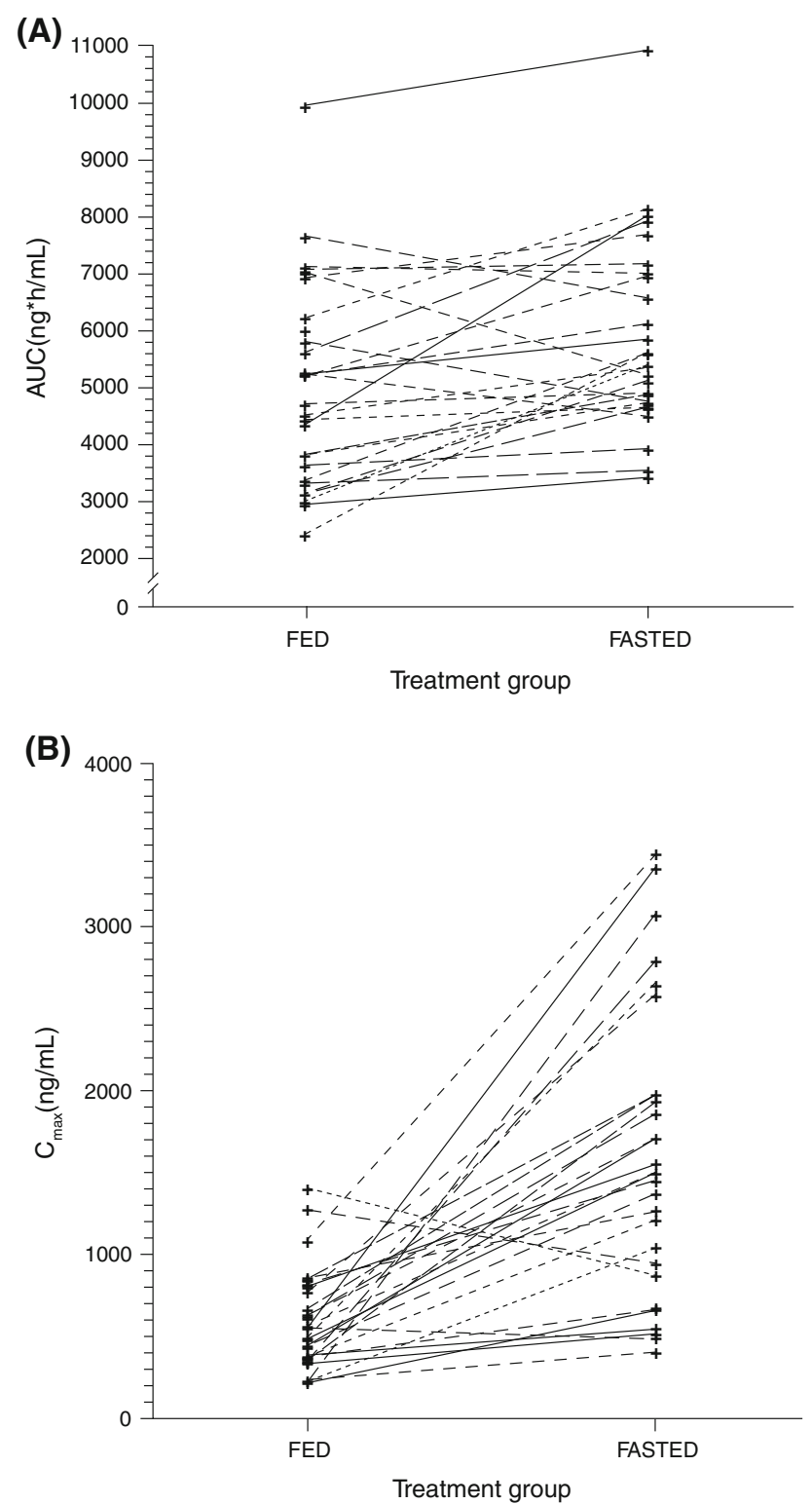

Fig. 2 AUC a and $\mathrm{C}_{\max } \mathbf{b}$ of selumetinib in fed and fasted state (PP population)

The exact mechanism for decreased selumetinib exposure in the presence of a high-fat meal is unknown and may be a result of saturation of drug dissolution in the intestinal fluids with an increased $\mathrm{pH}$. In the average person, the basal gastric secretion in the fasted stomach is estimated to be $300 \mathrm{~mL}$ and in the fasted small intestine about $500 \mathrm{~mL}$, and both can increase up to 5-fold after a high-fat meal [16, $18,20]$. On the other hand, fatty foods also stimulate bile secretion that can cause a significant rise in the $\mathrm{pH}$ of the proximal small bowel, the major site for selumetinib absorption $[16,21]$. The presence of more fluid in the gastrointestinal tract may actually improve the dissolution rate and can result in increased solubility due to increased wetting of the drug and increased micellar solubilization
Table 3 Most frequent AEs ( $\geq 10 \%$ of patients) in safety population

\begin{tabular}{ll}
\hline & $\begin{array}{l}\text { Number }(\%) \text { of patients } \\
\text { Total }(N=30)\end{array}$ \\
\hline Nausea & $18(60.0)$ \\
Rash & $16(53.3)$ \\
Diarrhea & $14(46.7)$ \\
Fatigue & $13(43.3)$ \\
Constipation & $11(36.7)$ \\
Dyspnoea & $10(33.3)$ \\
Vomiting & $9(30.0)$ \\
Abdominal pain & $8(26.7)$ \\
Dizziness & $8(26.7)$ \\
Edema peripheral & $7(23.3)$ \\
Anemia & $7(23.3)$ \\
Headache & $7(23.3)$
\end{tabular}

A patient can have one or more preferred term (PT) reported under a given system organ class

Patients with multiple events in the same PT are counted only once for that PT, within each period

Adverse events with an onset date during the 30 day follow-up are assigned to the period in which their last dose was administered

Table 4 SAE of $\geq$ CTC grade 3 reported in $\geq 2$ patients (safety population)

\begin{tabular}{ll}
\hline & $\begin{array}{l}\text { Number }(\%) \text { of patients } \\
\text { Total }(N=11)\end{array}$ \\
\hline Dyspnoea & $3(10.0)$ \\
Abdominal pain & $2(6.7)$ \\
Constipation & $2(6.7)$ \\
Vomiting & $2(6.7)$ \\
Anemia & $2(6.7)$ \\
\hline
\end{tabular}

$[16,22]$. However, since the food effect consisted of a decrease in selumetinib exposure, the effect of the raised $\mathrm{pH}$ in the proximal bowel might be of greater importance and help explain the observed decrease in absorption of selumetinib. Additionally, the selumetinib capsule has a hydroxypropylmethylcellulose coating and will tend to disintegrate slowly thus preventing early activation by gastric acid and probably resulting in a controlled release.

Delayed gastric emptying is believed to be the major mechanism for delayed absorption and could be the underlying mechanism for the delayed $t_{\max }$ of selumetinib given in the presence of food. The presence of food in the stomach can decrease the rate of presentation of the drug to the small intestine and delay the onset of absorption, especially for rapidly absorbed drugs $\left(\mathrm{C}_{\max }\right.$ achieved within $2 \mathrm{~h}$ in fasted state). For selumetinib in fasted state, 
$\mathrm{C}_{\max }$ was determined in a previous study at $1.5 \mathrm{~h}$ and therefore could fit in this category of drugs [15].

The study population was representative of the broader Phase I clinical population of patients with advanced solid malignancies in terms of baseline and demographic characteristics. As observed in previous selumetinib monotherapy studies $[14,15,19]$, the exploratory efficacy analysis of this study suggests that selumetinib is biologically active. During the study, two patients with melanoma had partial responses and four patients had stable disease; two patients had stable disease for $>100$ days (1 patient with colorectal cancer and 1 patient with skin/soft tissue cancer) and two patients had stable disease for $>50$ days ( 1 patient with prostate cancer and 1 patient with liver cancer). However, it should be noted that this study was not designed to investigate the efficacy of selumetinib, and tumor mutation status (e.g. BRAF V600E or KRAS) was not determined. Limited responses were to be expected from this advanced patient population who had progressed on multiple prior anti-cancer treatments, consistent with that seen in the Phase I trials.

The AE profile in this study was similar to that seen in previous selumetinib monotherapy studies $[14,15,19]$. The most common AEs included gastrointestinal AEs, dermatological AEs, and fatigue. The majority of these events occurred during BD dosing.

Food effect data are available with other MEK inhibitors that have been used clinically including CI-1040 (PD184352) and PD-0325901. CI-1040 was the first MEK inhibitor to enter the clinic, and in the Phase I study, dosing was initially performed in the morning on an empty stomach (after an overnight fast) [23]. It has been proposed that low aqueous solubility is responsible for the poor oral absorption of CI-1040 [23-25]; solubility of $<1 \mu \mathrm{g} / \mathrm{mL}$ in pH 6.5 phosphate buffer. Administration of CI-1040 at 800 and $1,600 \mathrm{mg}$ with a high-fat meal resulted in a 4.5 -fold increase in drug exposure of CI-1040 and a 5-fold increase of the metabolite PD-0184264. The MTD and recommended Phase II dose (RP2D) was determined at $800 \mathrm{mg}$ BID administered with food. The mechanism postulated for the observed increase in CI-1040 exposure in the presence of food was facilitated dissolution either by the fat in the food or because of increased presence of bile salts. This would be in line with preclinical dissolution data in which the addition of detergents to the dissolution media increased dissolution. Development of CI-1040 was discontinued in Phase II due to lack of efficacy. PD-0325901, another non-ATP competitive MEK inhibitor [26], is a derivative of CI-1040 with improved aqueous solubility of $190 \mu \mathrm{g} / \mathrm{mL}$ in $\mathrm{pH} 6.5$ phosphate buffer and is, like selumetinib, referred to as a second generation MEK inhibitor. PD-0325901 had a 50-fold higher potency than CI-1040, improved bioavailability, increased metabolic stability, and increased duration of target suppression (inhibition of ERK phosphorylation). In fasted conditions, PD-0325901 was absorbed rapidly with a $t_{\max }$ of $1 \mathrm{~h}$, and $\mathrm{C}_{\max }$ and AUC showed proportional increase at the tested doses; however, the effect of food on pharmacokinetics was not reported in the Phase I study. We would expect the food effect to be less than that for CI-1040 because of the improved solubility of PD-0325901. Due to lack of response and observed toxicity, development of PD-0325901 in Phase II was discontinued [27].

CH497655 (RO4987655) is a new MEK inhibitor and is currently being evaluated in a Phase I trial. Although a study has been performed in healthy volunteers, no information has been provided about the effect of food on the absorption. PK in healthy volunteers with doses up to $4 \mathrm{mg}$ showed rapid absorption (median $t_{\max }$ of $1 \mathrm{~h}$ ) and low variability [28].

In conclusion, PK parameters of orally administered drugs may be significantly altered in the presence of food due to changes in solubility, absorption and metabolism. The current study demonstrated that intake of a high-fat meal decreases the extent and rate of selumetinib absorption and suggests that a $75 \mathrm{mg}$ dose of selumetinib capsule formulation given twice a day has an acceptable safety profile in this advanced cancer population. It is recommended that for further clinical studies, selumetinib should be taken on an empty stomach.

Acknowledgments This study was sponsored by AstraZeneca.

Open Access This article is distributed under the terms of the Creative Commons Attribution Noncommercial License which permits any noncommercial use, distribution, and reproduction in any medium, provided the original author(s) and source are credited.

\section{References}

1. Chang L, Karin M (2001) Mammalian MAP kinase signalling cascades. Nature 410:37-40

2. Downward J (2003) Targeting RAS signalling pathways in cancer therapy. Nat Rev Cancer 3:11-22

3. Ramnath N, Adjei A (2007) Inhibitors of Raf kinase and MEK signaling. Update Cancer Ther 2:111-118. doi:10.1016/j.uct. 2007.10.001

4. Bonnet F, Vigneron M, Bensaude O, Dubois MF (1999) Transcription-independent phosphorylation of the RNA polymerase II C-terminal domain (CTD) involves ERK kinases (MEK1/2). Nucleic Acids Res 27:4399-4404

5. Weinstein-Oppenheimer CR, Blalock WL, Steelman LS, Chang F, McCubrey JA (2000) The Raf signal transduction cascade as a target for chemotherapeutic intervention in growth factorresponsive tumors. Pharmacol Ther 88:229-279

6. Davies BR, Logie A, McKay JS, Martin P, Steele S, Jenkins R, Cockerill M, Cartlidge S, Smith PD (2007) AZD6244 (ARRY142886), a potent inhibitor of mitogen-activated protein kinase/ extracellular signal-regulated kinase kinase $1 / 2$ kinases: 
mechanism of action in vivo, pharmacokinetic/pharmacodynamic relationship, and potential for combination in preclinical models. Mol Cancer Ther 6:2209-2219. doi:10.1158/1535-7163. MCT-07-0231

7. Yeh TC, Marsh V, Bernat BA, Ballard J, Colwell H, Evans RJ, Parry J, Smith D, Brandhuber BJ, Gross S et al (2007) Biological characterization of ARRY-142886 (AZD6244), a potent, highly selective mitogen-activated protein kinase kinase $1 / 2$ inhibitor. Clin Cancer Res 13:1576-1583. doi:10.1158/1078-0432.CCR06-1150

8. Haass NK, Sproesser K, Nguyen TK, Contractor R, Medina CA, Nathanson KL, Herlyn M, Smalley KS (2008) The mitogenactivated protein/extracellular signal-regulated kinase kinase inhibitor AZD6244 (ARRY-142886) induces growth arrest in melanoma cells and tumor regression when combined with docetaxel. Clin Cancer Res 14:230-239

9. Dummer R, Robert C, Chapman PB, Sosman JA, Middleton M, Bastholt L, Kemsley K, Cantarini MV, Morris C, Kirkwood JM (2008) AZD6244 (ARRY-142886) vs temozolomide (TMZ) in patients (pts) with advanced melanoma: an open-label, randomized, multicenter, phase II study. J Clin Oncol (Meeting Abstracts) 26:9033

10. Bodoky G, Timcheva C, Spigel DR, La Stella PJ, Ciuleanu TE, Pover G, Tebbutt NC (2011) A phase II open-label randomized study to assess the efficacy and safety of selumetinib (AZD6244 [ARRY-142886]) versus capecitabine in patients with advanced or metastatic pancreatic cancer who have failed first-line gemcitabine therapy. Invest New Drugs. (in press). doi:10.1007/ s10637-011-9687-4

11. Bennouna J, Lang I, Valladares-Ayerbes M, Boer K, Adenis A, Escudero P, Kim TY, Pover GM, Morris CD, Douillard JY (2010) A phase II, open-label, randomised study to assess the efficacy and safety of the MEK1/2 inhibitor AZD6244 (ARRY-142886) versus capecitabine monotherapy in patients with colorectal cancer who have failed one or two prior chemotherapeutic regimens. Invest New Drugs. doi:10.1007/s10637-010-9392-8

12. Hainsworth JD, Cebotaru CL, Kanarev V, Ciuleanu TE, Damyanov D, Stella P, Ganchev H, Pover G, Morris C, Tzekova V (2010) A phase II, open-label, randomized study to assess the efficacy and safety of AZD6244 (ARRY-142886) versus pemetrexed in patients with non-small cell lung cancer who have failed one or two prior chemotherapeutic regimens. J Thorac Oncol 5:1630-1636. doi:10.1097/JTO.0b013e3181e8b3a3

13. Bekaii-Saab T, Phelps MA, Li X, Saji M, Goff L, Kauh JS, O'Neil BH, Balsom S, Balint C, Liersemann R et al (2011) Multiinstitutional phase II study of selumetinib in patients with metastatic biliary cancers. J Clin Oncol 29:2357-2363. doi:10.1200/ JCO.2010.33.9473

14. Adjei AA, Cohen RB, Franklin W, Morris C, Wilson D, Molina JR, Hanson LJ, Gore L, Chow L, Leong S et al (2008) Phase I pharmacokinetic and pharmacodynamic study of the oral, smallmolecule mitogen-activated protein kinase kinase $1 / 2$ inhibitor AZD6244 (ARRY-142886) in patients with advanced cancers. J Clin Oncol 26:2139-2146. doi:10.1200/JCO.2007.14.4956

15. Banerji U, Camidge DR, Verheul HM, Agarwal R, Sarker D, Kaye SB, Desar IM, Timmer-Bonte JN, Eckhardt SG, Lewis KD et al (2010) The first-in-human study of the hydrogen sulfate (Hyd-sulfate) capsule of the MEK1/2 inhibitor AZD6244
(ARRY-142886): a phase I open-label multicenter trial in patients with advanced cancer. Clin Cancer Res 16:1613-1623. doi: 10.1158/1078-0432.CCR-09-2483

16. Custodio JM, Wu CY, Benet LZ (2008) Predicting drug disposition, absorption/elimination/transporter interplay and the role of food on drug absorption. Adv Drug Deliv Rev 60:717-733

17. Harris RZ, Jang GR, Tsunoda S (2003) Dietary effects on drug metabolism and transport. Clin Pharmacokinet 42:1071-1088

18. Singh BN (1999) Effects of food on clinical pharmacokinetics. Clin Pharmacokinet 37:213-255

19. Chow LQM, Eckhardt SG, Reid JM, Molina J, Hanson L, Piens J et al (2005) A first in human dose-ranging study to assess the pharmacokinetics, pharmacodynamics, and toxicities of the MEK inhibitor ARRY-142886 (AZD6244) in patients with advanced solid malignancies. AACR-NCI-EORTC International Conference on Molecular Targets and Cancer Therapeutics abstract $\mathrm{C} 162$

20. Welling PG (1996) Effects of food on drug absorption. Annu Rev Nutr 16:383-415

21. Lui CY, Amidon GL, Berardi RR, Fleisher D, Youngberg C, Dressman JB (1986) Comparison of gastrointestinal pH in dogs and humans: implications on the use of the beagle dog as a model for oral absorption in humans. J Pharm Sci 75:271-274

22. Charman WN, Porter CJ, Mithani S, Dressman JB (1997) Physiochemical and physiological mechanisms for the effects of food on drug absorption: the role of lipids and $\mathrm{pH}$. J Pharm Sci 86:269-282

23. LoRusso PM, Adjei AA, Varterasian M, Gadgeel S, Reid J, Mitchell DY, Hanson L, DeLuca P, Bruzek L, Piens J et al (2005) Phase I and pharmacodynamic study of the oral MEK inhibitor CI-1040 in patients with advanced malignancies. J Clin Oncol 23:5281-5293. doi:10.1200/JCO.2005.14.415

24. Rinehart J, Adjei AA, LoRusso PM, Waterhouse D, Hecht JR, Natale RB, Hamid O, Varterasian M, Asbury P, Kaldjian EP et al (2004) Multicenter phase II study of the oral MEK inhibitor, CI1040 , in patients with advanced non-small-cell lung, breast, colon, and pancreatic cancer. J Clin Oncol 22:4456-4462. doi: 10.1200/JCO.2004.01.185

25. Roberts PJ, Der CJ (2007) Targeting the Raf-MEK-ERK mitogen-activated protein kinase cascade for the treatment of cancer. Oncogene 26:3291-3310

26. LoRusso PM, Krishnamurthi SS, Rinehart JJ, Nabell LM, Malburg L, Chapman PB, DePrimo SE, Bentivegna S, Wilner KD, Tan W et al (2010) Phase I pharmacokinetic and pharmacodynamic study of the oral MAPK/ERK kinase inhibitor PD0325901 in patients with advanced cancers. Clin Cancer Res 16:1924-1937. doi:10.1158/1078-0432.CCR-09-1883

27. Haura EB, Ricart AD, Larson TG, Stella PJ, Bazhenova L, Miller VA, Cohen RB, Eisenberg PD, Selaru P, Wilner KD et al (2010) A phase II study of PD-0325901, an oral MEK inhibitor, in previously treated patients with advanced non-small cell lung cancer. Clin Cancer Res 16:2450-2457

28. Lee L, Niu H, Rueger R, Igawa Y, Deutsch J, Ishii N, Mu S, Sakamoto Y, Busse-Reid R, Gimmi C et al (2009) The safety, tolerability, pharmacokinetics, and pharmacodynamics of single oral doses of CH4987655 in healthy volunteers: target suppression using a biomarker. Clin Cancer Res 15:7368-7374 